\title{
Assessing the Validity of Asthma Associations for Eight Candidate Genes and Age at Diagnosis Effects
}

\author{
María Pino-Yanes ${ }^{1,20}$, Almudena Corrales ${ }^{1,2}$, José Cumplido ${ }^{3}$, Paloma Poza ${ }^{4}$, \\ Inmaculada Sánchez-Machín ${ }^{4}$, Anselmo Sánchez-Palacios ${ }^{5}$, Javier Figueroa ${ }^{5}$, \\ Orlando Acosta-Fernández ${ }^{6}$, Nisa Buset ${ }^{7}$, José Carlos García-Robaina ${ }^{8}$, Mariano Hernández ${ }^{9}$, \\ Jesús Villar ${ }^{1,7,10}$, Teresa Carrillo ${ }^{3}$, Carlos Flores ${ }^{1,2,9 *}$
}

1 Centro de Investigación Biomédica en Red de Enfermedades Respiratorias (CIBERES), Instituto de Salud Carlos III, Madrid, Spain, 2 Research Unit, Hospital Universitario Nuestra Señora de Candelaria, Santa Cruz de Tenerife, Spain, 3 Allergy Unit, Hospital Universitario Dr. Negrín, Las Palmas de Gran Canaria, Spain, 4 Allergy Management Unit, Hospital del Torax (Ofra), Santa Cruz de Tenerife, Spain, 5 Allergy Unit, Hospital Universitario Insular de Gran Canaria, Las Palmas de Gran Canaria, Spain, 6 Neumology Unit, Hospital Universitario de Canarias, San Cristóbal de La Laguna, Spain, 7 Research Unit, Hospital Universitario Dr. Negrin, Las Palmas de Gran Canaria, Spain, 8 Allergy Unit, Hospital Universitario Nuestra Señora de Candelaria, Santa Cruz de Tenerife, Spain, 9 Applied Genomics Group (G2A), Genetics Laboratory, Instituto Universitario de Enfermedades Tropicales y Salud Pública de Canarias, Universidad de La Laguna, San Cristóbal de La Laguna, Spain, 10 Keenan Research Center, St. Michael' Hospital, Toronto, Ontario, Canada

\begin{abstract}
Background: Before the advent of genome-wide association studies (GWAS), ADAM33, ADRB2, CD14, MS4A2 (alias FCER1B), $I L 13, I L 4, I L 4 R$, and TNF constituted the most replicated non-HLA candidate genes with asthma and related traits. However, except for the IL13-IL4 region, none of these genes have been found in close proximity of genome-wide significant hits among GWAS for asthma or related traits. Here we aimed to assess the reproducibility of these asthma associations and to test if associations were more evident considering the effect of age at diagnosis.
\end{abstract}

Methodology/Principal Findings: We systematically evaluated 286 common single nucleotide polymorphisms (SNPs) of these 8 genes in a sample of 1,865 unrelated Spanish individuals (606 asthmatics and 1,259 controls). We found that variants at MS4A2, IL4R and ADAM33 genes demonstrated varying association effects with the age at diagnosis of asthma, with 10 SNPs showing study-wise significance after the multiple comparison adjustment. In addition, in silico replication with GWAS data supported the association of IL4R.

Conclusions/Significance: Our results support the important role of MS4A2, IL4R and ADAM33 genes in asthma and/or atopy susceptibility. However, additional studies in larger samples sets are needed to firmly implicate these genes in asthma susceptibility, and also to identify the causal variation underlying the associations found.

Citation: Pino-Yanes M, Corrales A, Cumplido J, Poza P, Sánchez-Machín I, et al. (2013) Assessing the Validity of Asthma Associations for Eight Candidate Genes and Age at Diagnosis Effects. PLoS ONE 8(9): e73157. doi:10.1371/journal.pone.0073157

Editor: Gualtiero I. Colombo, Centro Cardiologico Monzino IRCCS, Italy

Received February 13, 2013; Accepted July 18, 2013; Published September 9, 2013

Copyright: (c) 2013 Pino-Yanes et al. This is an open-access article distributed under the terms of the Creative Commons Attribution License, which permits unrestricted use, distribution, and reproduction in any medium, provided the original author and source are credited.

Funding: Funded by grants from the Carlos III Health Institute (http://www.isciii.es) and cofounded by the European Regional Development Fund (FEDER) "A way of making Europe": PI081383 and EMER07/001, and by a grant from Fundación Canaria de Investigación y Salud FUNCIS 27/07 (http://www.funcis.org) to CF. MPY was funded by a postdoctoral fellowship from Fundación Ramón Areces (http://www.fundacionareces.es). The funders had no role in study design, data collection and analysis, decision to publish, or preparation of the manuscript.

Competing Interests: The authors have declared that no competing interests exist.

*E-mail: cflores@ull.edu.es

a Current address: Department of Medicine, University of California San Francisco, San Francisco, California, United States of America

\section{Introduction}

Asthma is a complex respiratory disease characterized by chronic inflammation of the airways and frequently associated with atopy, pulmonary obstruction and bronchial hyper-responsiveness against a diversity of stimulus [1]. Its prevalence varies widely among different populations around the world (1-18\%) [2]. Familiar clustering [3], twin studies [4], and genetic studies [5,6] support an important genetic component of the disease, with an estimated heritability of $60 \%[7]$.

Before the advent of genome-wide association studies (GWAS) [8], almost a thousand candidate-gene association studies for asthma and related traits were published [9]. Considering the gene as the unit of replication and using a broad definition for asthma, Ober \& Hoffjan [5] elegantly summarized the accumulated evidence for candidate-gene association studies from the literature by assessing the consistency of findings [10]. This yielded a ranking of candidate genes based on the number of positive associations between any polymorphism and any asthma trait [5]. As a result, eight non-HLA genes were put forward among the most replicated (in $>10$ independent studies) and, therefore, these genes were suggested as firm candidates for asthma susceptibility [5]. Four of these genes were located in the linked region for asthma on chromosome 5q: interleukin (IL) 4 (IL4), IL13, CD14 and the $\beta 2-$ adrenergic receptor (ADRB2); one gene was located in the linked region 6p21: the tumor necrosis factor $(T \mathcal{N} F)$; one was the first 
positionally cloned asthma gene, ADAM metallopeptidase domain 33 (ADAM33); another was the gene encoding the $\alpha$ chain of the IL-4 and IL-13 receptors (IL4R); and finally, the gene encoding the IgE Fc receptor beta-subunit (MS4A2, alias FCER1B). However, most of them were assessed in studies with limited sample sizes averaging $\approx 200$ individuals per group [5,11], and lacked of a systematical analysis in candidate-gene studies by surveying more than few variants (for example, by using tagging SNPs [tSNPs]). To date, despite the fact that more than ten GWAS of asthma have been published, none of these eight firm candidates have been replicated at genome wide significance, nor have been found in close proximity of GWAS hits, except for the IL13-IL4 region $[8,12-21]$.

Asthma is clinically recognized as an amalgam of several distinct phenotypes [22,23], which blur the complex genetic architecture underlying the disease susceptibility. Among these phenotypes, the age-at-onset of asthma could differentiate asthmatic groups, so that genetic variants might inconsistently associate with childhood and later-onset disease $[17,24,25]$. Motivated by this evidence, here we aimed to assess the reliability of asthma associations for the eight most replicated non- $H L A$ asthma candidate genes and to explore whether effects of risk alleles varied with the disease age at diagnosis.

\section{Methods}

\section{Ethics statement}

This study was approved by the External Scientific Committee and Advisory Committee of Experts on ethical, economic, environmental, legal and social affairs at the National Bank and the Ethics Committee of Hospital Universitario NS de Candelaria and Hospital Universitario Doctor Negrín. Written informed consent was obtained from all subjects or appropriate surrogates on the behalf of participants under the age of 18 .

\section{Study subjects}

This study was conducted using a case-control design of 1,878 DNA samples from unrelated individuals, all reporting at least two generations of Spanish descent. Sample details have been described elsewhere [24]. In brief, cases included 607 asthmatic patients aged $>5$ years and diagnosed by physicians following the Global Initiative for Asthma (GINA) guidelines for diagnosis and classification of asthma severity (http://www.ginasthma.com). These samples were collected and characterized for allergic and asthmatic symptoms in Respiratory Medicine and Allergy Departments, as part of the Genetics of Asthma study (GOA) in the Spanish population. Among cases, atopy was defined by the evidence of allergic sensitization to known allergens, reflected by either a positive skin prick test [SPT] or the specific IgE to one or more known allergens in the serum. For simplicity, those cases that had asthma and also atopy will be referred as atopic asthmatics, although we ignored whether or not allergen exposures lead to the asthma symptoms of these patients. Further sample details can be found in Text $\mathrm{S} 1$ and in Table S1.

Control group consisted of 1,271 DNA samples from adults selfreporting no personal or familiar medical history of allergic or pulmonary diseases recruited from the Spanish National DNA Biobank. These were collected from branches of the National Blood Service from unrelated individuals residing in Spain. After signed informed consent, by means of personal interviews, each donor was asked to declare general health status, physic activity, commonly used transportation, nutrition habits, type of work and qualification, demographics, tobacco smoke, alcohol consumption, genealogical information, residence and mother tongue, and personal and familial history of diseases. See http://www. bancoadn.org for further information. In addition to the criteria of the Spanish National DNA Biobank to define healthy controls, we added three more criteria to select the controls for this study: 1) Self-reported Spanish ancestry based on having at least two generations of ancestors born in Spain; 2) Complete data on personal and familiar history of disease recorded in the questionnaire, smoking status, place of origin, and area of residence; 3) Absence of self-reported personal or familiar history of pulmonary or allergic disease. Further sample details can be found in Text S1 and in Table S1.

\section{Selection of tagging SNPs}

Tagging SNPs (tSNPs) were selected by means of TagIT 3.03 [26], using available re-sequencing data from European samples from different sources (Table 1 and Table S2). The IL13 and IL4 genes, which lie in close proximity, were considered as a single region. Similarly, given the strong linkage disequilibrium (LD) between LTA and TNF genes [27], common variants of the LTA gene were also tagged and jointly analyzed with $T \mathcal{N} F$. See Text $\mathrm{S} 1$ and Table S2 for further details.

\section{Assessment of population stratification}

To reduce the risk for false positives due to major population stratification effects, a total of 83 European ancestry informative markers (termed EuroAIMs) were determined in case and control samples. These EuroAIMs allowed to correct for major differences in Spanish populations due to the North African genetic influences observed in this population, with a mean value of 5-9\% for mainland populations and 16-20\% for Canary Islanders [28]. A principal component analysis (PCA) based on these genetic markers was used to derive the ancestry estimates in cases and controls as scores of the first principal component (PC1), by means of EIGENSOFT [29]. A full list of EuroAIMs used and the genotyping procedures have been detailed elsewhere $[24,28]$.

\section{Genotyping}

Genotyping was conducted using the PLEX $^{\circledR}$ Gold assay on MassARRAY ${ }^{\circledR}$ system (Sequenom Inc., San Diego, CA) by the Spanish National Genotyping Center, Santiago de Compostela Node (CeGen, http://www.cegen.org). Briefly, iPLEX ${ }^{\circledR}$ assays were scanned by MALDI-TOF mass spectrometry and individual SNP genotype calls were automatically generated using Sequenom TYPER 3.4 ${ }^{\circledR}$ software (Sequenom Inc.). Samples from the Coriell Institute for Medical Research (http://www.coriell.org) were included on each SpectroCHIP ${ }^{\circledR}$ (Sequenom Inc.) to test allele calling reliability samples of this platform. The SNPs that gave poor quality data on this platform were finally determined at the Hospital Universitario N. S. de Candelaria using either SNaPshot ${ }^{\circledR}$ Multiplex kit reactions (Applied Biosystems, Foster City, CA) or KASPar SNP Genotyping System assays (KBiosciences, Hertfordshire, UK). Genotyping was blind to the disease status and $\approx 6 \%$ of the samples were genotyped in duplicate to monitor genotyping quality. See Text S1 for further details.

\section{Statistical analysis}

Glinical and demographical data were analyzed by means of the $\chi^{2}$-test and the Mann-Whitney U-test using $\mathrm{R}$ version 2.15 [30]. Departures from Hardy-Weinberg equilibrium (HWE) were evaluated separately for cases and controls using an exact test [31], by means of a custom script for STATISTICA (StatSoft Inc., Tulsa, OK) [32]. However, as deviations in cases have been considered a symptom of disease association [33-35], only those 
Table 1. Summary information used for the selection of tagging SNPs (tSNPs) on the candidate genes.

\begin{tabular}{|c|c|c|c|c|c|c|c|c|}
\hline Gene & Chr. (Mb) & Size (kb) & $\begin{array}{l}\text { Covered } \\
\text { region }(\mathbf{k b})\end{array}$ & Data sources & $\begin{array}{l}\text { Selected } \\
\text { tSNPs }\end{array}$ & Monomorphic & $\begin{array}{l}\text { Final } \\
\text { tSNPs }\end{array}$ & $\begin{array}{l}\text { Final } \\
\text { haplotype } \\
r^{2}\end{array}$ \\
\hline IL13-IL4 & 5q31.1 (132.0) & 12 & 29.0 & SeattleSNPs ${ }^{a}$ & 10 & 0 & 10 & 1.00 \\
\hline$C D 14$ & 5q31.1 (140.0) & 2 & 7.0 & $\begin{array}{l}\text { Innate } \\
\text { Immunity }^{b}\end{array}$ & 6 & 1 & 5 & 1.00 \\
\hline ADRB2 & $5 q 31(148.2)$ & 2 & 9.5 & SeattleSNPs ${ }^{a}$ & 8 & 1 & 7 & 1.00 \\
\hline TNF-LTA & 6p21.3 (31.5) & 6 & 9.3 & SeattleSNPs ${ }^{a}$ & 11 & 0 & 11 & 0.85 \\
\hline MS4A2 & $11 q 13(59.9)$ & 10 & 15.3 & HapMap/ T1D & 7 & 0 & 7 & 0.97 \\
\hline IL4R & 16p12.1 (27.3) & 51 & 56.0 & SeattleSNPs ${ }^{a}$ & 21 & 0 & 21 & 0.92 \\
\hline ADAM33 & 20p13 (3.6) & 14 & 15.2 & $E G P^{d}$ & 19 & 4 & 15 & 1.00 \\
\hline Total & & 97 & 141.3 & & 82 & 6 & 76 & \\
\hline
\end{tabular}

${ }^{\mathrm{a}}$ The National Heart Lung and Blood Institute's (NHLBI) Programs for Genomic Applications (http://pga.gs.washington.edu).

${ }^{\mathrm{b}}$ The Inate Immunity NHLBI Program for PGA (https://regepi.bwh.harvard.edu/IIPGA2).

'HapMap phase 2 (http://hapmap.ncbi.nlm.nih.gov) and data from 96 type 1 diabetes individuals [28].

dThe NIEHS Environmental Genome Project (http://egp.gs.washington.edu).

doi:10.1371/journal.pone.0073157.t001

tSNPs deviating significantly from HWE in the control group were filtered out from further analyses (threshold $p$-value $=7.0 \mathrm{E}-04$ after considering the multiple comparisons performed). Individual tSNP associations were tested under an additive model by means of regression analysis with SNPassoc [36]. For that, PG1 scores were included as a covariate in regression models to adjust associations for population stratification, and allele effects were estimated as odds ratios (ORs) with 95\% confidence intervals (CIs).

Additionally, MaCH 1.0 [37] was used to impute untyped SNPs with data from 380 European individuals deposited in The 1000 Genomes Project (1KGP), May 2011 version [38]. Association testing was performed using Mach2dat [37] adjusting for the PG1 scores. This analysis was conducted using allele dosages for those SNPs showing MAF $\geq 10 \%$ and Rsq $>0.3$, ensuring that all SNPs considered for association testing were accurately imputed (with $>90 \%$ of SNPs having Rsq $>0.8$, and with a mean Rsq across all imputed SNPs of 0.95 [IQR: 0.91-0.97]) (Table S3).

For each gene by separate, a conditional regression-based analysis was used to point out the independent association signals of each locus by including all SNPs associated at nominal significance. We then tested if association tests of the SNPs that represented nominal independent associations within each gene improved considering age-at-onset-varying effects, by implementing a sequential addition (SA) of cases [39]. For that, the age at diagnosis was utilized as a proxy for the age-at-onset of the disease, which was not recorded for most patients, and cases were grouped in categories of quartiles of age $(14[n=155], 26[n=291], 39$ $[\mathrm{n}=427]$, and 82 years $[\mathrm{n}=606])$. The age at diagnosis cutoff obtained was next used to select a sub-sample of cases for which associations were tested again, both for tSNPs and imputed SNPs. LD patterns and regional association results were represented using LocusZoom 1.1 based on LD data from hglo deposited by 1KGP [40].

To judge the significance of SNP associations in the context of the multiple comparisons performed, a false discovery rate (FDR) was calculated using QVALUE [41]. A FDR threshold of 5\% (pvalue $\leq 0.0012)$ was established to declare study-wise significance to limit the expected proportion of false positives incurred in the study when a particular individual SNP test was called significant. This was assessed considering altogether the $p$-values from all SNPs analyzed, both genotyped and imputed, the tests from the
SA of cases to obtain the age cutoff at which the allele effects were largest, and all the comparisons performed (i.e. associations with asthma, atopic asthma, and age-of-onset before the cutoffs). Functional annotation of associated SNPs was carried out using the software HaploReg [42].

\section{Results}

A total of 13 samples (1 case and 12 controls) were excluded from the analyses because of genotype quality (completion rate $<90 \%$ ). Out of the initial set of $82 \mathrm{tSNPs}$, 6 were found monomorphic (rs5744440, rs35684381, rs597040, rs8124875, rs614971 and rs17548816) by using both iPLEX $^{\circledR}$ and an alternative genotyping method (see the Supplementary methods in Text S1). Only one tSNP (rs12361312 at MS4A2) deviated significantly from HWE expectations in the control group and was discarded from further analyses (Table S2). Therefore, a total of 75 tSNPs, which maintained an adequate coverage for all genes $\left(r^{2} \geq 0.85\right)$, and 211 imputed SNPs were considered for association studies in 1,865 samples (606 cases and 1,259 controls) (Table S3). The mean completion rate among the $75 \mathrm{tSNPs}$ was $98.5 \%\left(\mathrm{P}_{25}-\right.$ $\mathrm{P}_{75}=98.7-100.0 \%$ ), and the estimated overall genotype discordance rate among duplicated samples was $0.30 \%$ (95\% CI = $0.08 \%-1.09 \%)$.

Association testing revealed a total of 35 SNPs (16 tSNPs and 19 imputed) that were associated with either asthma or atopic asthma at nominal significance, although these were not considered significant in the context of the multiple comparisons ( $p$-values $>0.0012$ ) (Table S3). Based on the premise that incorporating the age-at-onset in the analyses might increase the power to detect association $[24,25]$, we next used SA of asthma patients to estimate the age at diagnosis cutoff maximizing allele effects. For that, among the 35 SNPs that reached nominal significance, we first excluded the redundant SNPs from each gene using conditional logistic regressions for asthma or atopic asthma. We identified the following SNPs as the ones showing independent nominal associations: rs1800925 (-1112 C/T) in IL13-IL4 (only for atopic asthma), rs2071590 in LTA-TNF (only for asthma), rs569108 (Gly237Glu) in MS4A2, and rs 1805015 (Ser478Pro) in IL4R (both for asthma and atopic asthma) and rs2787095 in ADAM33 (only for asthma) (data not shown). These 5 SNPs represented independent associations for each gene and coincidentally, these 
5 SNPs had been associated with asthma or related traits in previous studies, but in this study we extended their association to a Southwestern European population with noticeable North African influences.

SA did not show any age at diagnosis cutoff that significantly maximized the association of rs1800925 (in IL13-IL4) with atopic asthma (lowest $p$-perm $=0.063$ ). In contrast, SA revealed allele effects peaking at the same age at diagnosis, 39 years (number of cases =427), for SNPs from MS4A2 and IL4R: rs569108 in MS4A2 (p-perm $=0.005)$, and rs1805015 in IL4R (p-perm $=0.001)$. However, SA revealed allele effects peaking at a different age at diagnosis for the SNPs from the other two genes: rs2071590 in $L T A-T N F$ showing a maximum at 26 years $(\mathrm{p}$-perm $=0.002$, number of cases $=291$ ), and rs2787095 in ADAM33 with a maximum at 14 years $(\mathrm{p}$-perm $=3.0 \mathrm{E}-04$, number of cases $=$ 155). The results obtained from the SA analyses using the quartiles of the distribution of the age at diagnosis were equivalent to those obtained using it as a continuous variable (data not shown).

Testing associations on the sub-sample of cases with the age at diagnosis of asthma before the maxima determined by SA for each gene revealed 18 additional SNPs reaching nominal significance (Table S3). Five of these SNPs $(0.013 \leq$ p-value $\leq 0.050)$ were located in IL4R gene and only one of them constituted a positive finding in previous studies. After conditioning these new associations from IL4R to the SNP rs1805015, only one SNP showed independent association (rs3024676, p-value $=0.021$ ). The remaining 13 were all SNPs from ADAM33 (3.8E-5 $\leq$ p-value $\leq 0.039$ ), and 6 of them have been associated in at least one previous study (Table S3 and Figure 1). After adjusting the association of these 13 SNPs in ADAM33 that emerged with the age at diagnosis cutoff for the SNP rs2787095, 7 SNPs (rs2787093, rs628965, rs628977, rs630712, rs598418, rs2853209, and rs603112) resulted independently associated from this SNP $(0.012 \leq \mathrm{p}$-value $\leq 0.048)$. Therefore, the advantages of taking into account the age at diagnosis varying effects for replication studies in asthma were clearly evidenced in $A D A M 33$, a gene for which SNP-level replications are scarce in the literature $[5,43]$. Otherwise, we would have missed $>50 \%$ of SNPs of this gene that showed association in previous studies. For the LTA-TNF and MS4A2 genes, we only observed subtle increases of effect sizes for the SNPs that were revealed in our previous analyses, but did not evidence more SNPs reaching nominal significance (Table S3 and Figure 1). After a global FDR assessment accounting for all comparisons performed, only $10 \mathrm{SNPs}$ in MS4A2, IL4R and ADAM33 genes showed an FDR $<5 \%$, which were considered associated at study-wise significance (Table 2). Among these, 7 SNPs were identified to be functional, as they were either predicted to cause missense changes in the protein encoded or had empirically demonstrated regulatory roles as deduced from ENCODE project experimental data [42] (Table S4).

In order to provide evidence for replication at these loci, we accessed the GABRIEL data, the largest GWAS meta-analysis in asthma performed in Europeans [17]. There, we were able to allocate 11 out of the 51 SNPs that reached nominal significance with asthma in our study (Table S5). Only the SNP rs1805012, located in IL4R, demonstrated in silico replication in GABRIEL $(p=5.7 \mathrm{E}-04)$, showing the same direction of effects as in our study.

\section{Discussion}

In this study, we have comprehensively analyzed the association of 286 common variants of eight candidate genes with asthma and atopic asthma in a case-control Spanish sample and found associations for $10 \mathrm{SNPs}$ in three of them (MS4A2, IL4R and
ADAM33) after considering all tests performed. We additionally provided in silico replication for ILAR with GWAS data from the GABRIEL study.

It is well known that the age-at-onset of asthma is associated with different phenotypic characteristics [44], and it has recently evidenced that age-varying genetic associations can cause nonreplication and, consequently, lead to missing important genetic associations [45]. Therefore, here we re-evaluated the association of these genes by restricting the analysis to case subjects with an age at diagnosis of asthma before a cutoff that maximized allele effects of replicated variants. This allowed us to verify that association improved for certain genes, such as ADAM33, as recently supported for other firm candidates [25,46,47], and also to gain insight in the genetic complexity of asthma associations at these candidate genes. Intriguingly, many of their effects peaked in the range of age at diagnosis between 20 and 45 years, coincidental with the age range with the maximum expression of the disease $[48,49]$. It remains to be solved whether or not true biological mechanisms underlie this and previous observations $[17,25,46]$. Nevertheless, our results suggest that it will be worth considering the disease age at diagnosis in further studies, as well as in the research of improved asthma treatment and prevention.

To identify firm susceptibility genes and understand the biological processes underlying the development of the disease, replication in independent well-powered studies is essential, regardless of whether the first evidence of association was provided by a GWAS study or a candidate gene survey [50]. Besides, replication efforts allow testing the generalizability of findings in other populations, and discovering novel genetic loci contributing to phenotypic trait variability [51]. Particularly, testing the associations in populations of recent African ancestry will likely improve the detection of new risk variants [52], as they may offer the opportunity to refine the signal or to allocate the causal variants [53]. Our study aligns with these considerations, as it was performed in a population with sizeable North African genetic influences [28,54], and with a sample size representing a substantially larger population of cases $(>97 \%)$ than the vast majority of prior published case-control studies of these genes in unrelated individuals, although still far from optimal to detect weak effects.

Under a simplistic scenario assuming complete LD of associated SNPs with causal variants, the analyzed sample size provided a $70 \%$ power to detect a minimum risk of 1.45 for a risk allele frequency of $45 \%$ with a two-sided $p=0.0012$ significance level for the primary outcome (asthma), and ranged from 14.6\% to $52.6 \%$ for the analyses in subset of cases with atopic asthma and asthma before the age at diagnosis cutoff (Table S6). We acknowledge that risk effects of this range are in the upper bound of those expected for common variants in complex traits [55], which may have contributed to our failure to detect associations for some of the genes tested. Alternatively, our failure to find associations may possibly be attributed to: i) Our impossibility to test their association with more relevant traits or patient sub-samples (e.g. asthma drug responses [56,57], environmental exposures [58]); ii) The use of controls self-reporting no personal or familiar history of pulmonary or allergic disease, but without a disease confirmation based on a clinical characterization (e.g. lung function measurements, SPT or specific IgE testing); iii) The lack of a true association with asthma susceptibility, as has been suggested for particularly relevant variants by meta-analyses [56]. Whichever is correct, a recently published study with on a similar sample size showed positive and negative association results fully congruent with ours [59]. In support of our results, we were able to replicate in silico the association of a SNP in IL4R in the largest GWAS study 
a

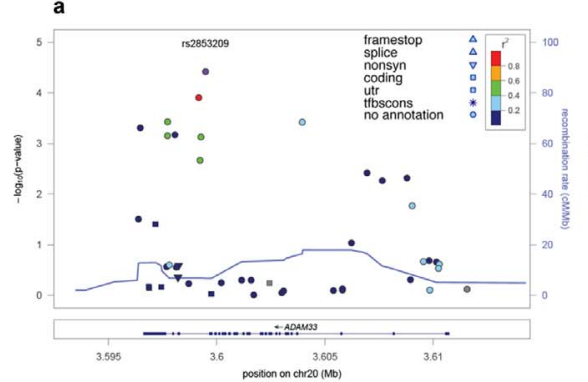

b

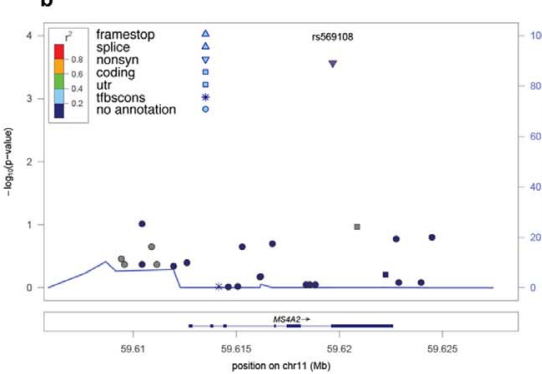

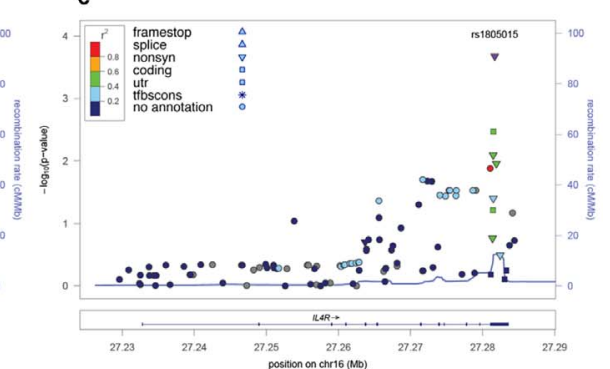

Figure 1. $P$-values of association by chromosome position with A) asthma $\leq 14$ years for $A D A M 33$, B) asthma $\leq 39$ years for $M S 4 A 2$ and, C) asthma $\leq 39$ years for IL4R. $P$-values are expressed in - $\log _{10}$ scale. The SNP number shown on the plot denotes the result for the most significant SNP for each gene and the results for the remaining were color coded to reflect their LD with this SNP based on pairwise $r^{2}$ values from the 1KGP. Estimated recombination rates (from 1KGP) were also plotted on the right axis to reflect the local LD structure.

doi:10.1371/journal.pone.0073157.g001

published to date that included more than 25,000 Europeans [17]. This SNP from $I L 4 R$, as well as few others from the same gene that were found associated in our study (rs1801275, rs1805015, and rs3024676), also demonstrated congruent effects and significant association in a recent GWAS of total IgE levels [60]. This evidence supports that, despite the enormous efforts to disentangle asthma genes such as those entailed by the GABRIEL study [17] or the EVE consortium [19], many more asthma susceptibility genes awaits its discovery.

Some recent replication studies focusing on candidate genes have utilized available arrays for genome wide genotyping [61-63] where common variants of many key asthma candidate genes could be insufficiently covered. In this respect, Michel et al. [59] indicated that only $37 \%$ of the previously associated SNPs from 14 candidate genes were captured by the array utilized by the same authors on the first GWAS for asthma and, surprisingly, not a single SNP from key asthma genes such as ADAM33, IL4 and CD14 was contained in their array [8]. Only after extending the study by further genotyping (and by imputation) on the same samples of their GWAS, these authors were able to consistently replicate many of the biological candidates that were missing from their GWAS [59]. We confirmed that the coverage of published
GWAS for asthma performed in European populations to date has been insufficient for ADAM33 (<30\%), even in a best-case scenario using the HapMap phase 2 data as a reference for comparisons (Table S7). If array comparisons were made against the 1KGP sequencing data [38], the coverage would be even lower (Table S7). Besides, it is worth noting that the estimated coverage of these genes might be inflated, as these were implicitly derived for HapMap CEU data and the same data was used to inform the SNP contents of the array, and we have assumed that the $100 \%$ of SNPs contained in the array were successfully genotyped. Effects similar to those related to the age-of-onset of asthma, exceptionally explored [17], could have also contributed to find no association for the genes explored here in the published GWAS for asthma.

In conclusion, here we found the association of 10 common variants in three biological candidate genes (MS4A2, IL4R and $A D A M 33)$ that attained study-wise significance, and one of them was also supported by in silico replication in GWAS data. Therefore, we provided independent support for their role as risk factors for the amalgam of asthma phenotypes. Moreover, our results evidenced the genetic complexity at some of these susceptibility loci and the importance of considering age-at-onset effects. Given the low statistical power of the present study,

Table 2. Association summary of the 10 SNPs that resulted significantly associated with asthma after adjustments for the multiple comparisons.

\begin{tabular}{|c|c|c|c|c|c|c|c|c|}
\hline Gene & rs\# & Position $^{a}$ & Comparison & $\begin{array}{l}\text { Allele1/ } \\
\text { Allele2 }\end{array}$ & $\begin{array}{l}\text { Frecuency } \\
\text { controls }^{\mathbf{b}}\end{array}$ & $\begin{array}{l}\text { Frecuency } \\
\text { cases }^{b}\end{array}$ & OR $(95 \% \mathrm{CI})^{\mathrm{b}}$ & $p$-value \\
\hline MS4A2 & rs569108 & 59863104 & Asthma diagnosed $\leq 39$ & $A / G$ & 0.961 & 0.985 & $2.45(1.39-4.33)$ & $2.7 \mathrm{E}-04^{\mathrm{C}}$ \\
\hline$I L 4 R$ & rs1805015 & 27374180 & Asthma diagnosed $\leq 39$ & $\mathrm{~T} / \mathrm{C}$ & 0.809 & 0.864 & $1.45(1.17-1.80)$ & $2.1 \mathrm{E}-04^{\mathrm{C}}$ \\
\hline \multirow[t]{8}{*}{ ADAM33 } & rs2787093 & 3648462 & Asthma diagnosed $\leq 14$ & $\mathrm{~T} / \mathrm{C}$ & 0.890 & 0.822 & $0.56(0.40-0.78)$ & $4.9 \mathrm{E}-04$ \\
\hline & rs628965 & 3649713 & Asthma diagnosed $\leq 14$ & $\mathrm{G} / \mathrm{A}$ & 0.619 & 0.523 & $0.65(0.50-0.84)$ & $1.0 \mathrm{E}-04^{\mathrm{c}}$ \\
\hline & rs628977 & 3649721 & Asthma diagnosed $\leq 14$ & $\mathrm{C} / \mathrm{T}$ & 0.617 & 0.516 & $0.66(0.51-0.85)$ & $3.7 \mathrm{E}-04^{\mathrm{c}}$ \\
\hline & rs630712 & 3650066 & Asthma diagnosed $\leq 14$ & $\mathrm{~A} / \mathrm{C}$ & 0.892 & 0.826 & $0.57(0.41-0.80)$ & $1.0 \mathrm{E}-04$ \\
\hline & rs597980 & 3651165 & Asthma diagnosed $\leq 14$ & $A / G$ & 0.440 & 0.326 & $0.60(0.46-0.79)$ & $1.2 \mathrm{E}-04^{\mathrm{c}}$ \\
\hline & rs598418 & 3651269 & Asthma diagnosed $\leq 14$ & $A / G$ & 0.618 & 0.523 & $0.65(0.50-0.84)$ & $1.0 \mathrm{E}-04^{\mathrm{c}}$ \\
\hline & rs2853209 & 3651472 & Asthma diagnosed $\leq 14$ & $\mathrm{~A} / \mathrm{T}$ & 0.482 & 0.362 & $0.58(0.45-0.75)$ & $3.8 \mathrm{E}-05^{\mathrm{C}}$ \\
\hline & rs2787095 & 3655943 & Asthma diagnosed $\leq 14$ & $\mathrm{C} / \mathrm{G}$ & 0.584 & 0.530 & $1.51(1.19-1.91)$ & $3.8 \mathrm{E}-04^{\mathrm{C}}$ \\
\hline
\end{tabular}

tSNPs are underlined.

according to NCBI build 36.3 .

${ }^{\mathrm{b}}$ Computed for allele 1.

'SNPs associated in previous studies.

doi:10.1371/journal.pone.0073157.t002 
particularly limited in the case subset analyses when considering the age at diagnosis, further studies will be needed to identify causal variants and to unravel if these genes are truly associated with asthma, with atopy or with both.

\section{Supporting Information}

Table S1 Relevant demographic and clinical features of GOA samples.

(DOC)

Table S2 Information, completion rates and HardyWeinberg equilibrium (HWE) $p$-values for the tSNPs. (DOC)

Table S3 Association summary of SNPs with asthma, atopic asthma and asthma with age at diagnosis before the cutoff demonstrating the largest effects.

(DOC)

Table S4 Functional annotation of the 10 associated SNPs.

(DOC)

Table S5 In silico replication of the associated SNPs contained in the GABRIEL study.

(DOC)

Table S6 Sample sizes and statistical power for each analysis performed in a subset of cases.

\section{References}

1. Ober C (2005) Perspectives on the past decade of asthma genetics. J Allergy Clin Immunol 116: 274-278.

2. Braman SS (2006) The global burden of asthma. Chest 130: S4-12.

3. Holberg CJ, Halonen M, Wright AL, Martinez FD (1999) Familial aggregation and segregation analysis of eosinophil levels. Am J Respir Crit Care Med 160: 1604-1610.

4. Thomsen SF, van der Sluis S, Kyvik KO, Skytthe A, Backer V (2010) Estimates of asthma heritability in a large twin sample. Clin Exp Allergy 40: 1054-1061.

5. Ober C, Hoffjan S (2006) Asthma genetics 2006: the long and winding road to gene discovery. Genes Immun 7: 95-100.

6. Bouzigon E, Forabosco P, Koppelman GH, Cookson WO, Dizier MH, et al. (2010) Meta-analysis of 20 genome-wide linkage studies evidenced new regions linked to asthma and atopy. Eur J Hum Genet 18: 700-706.

7. Duffy DL, Martin NG, Battistutta D, Hopper JL, Mathews JD (1990) Genetics of asthma and hay fever in Australian twins. Am Rev Respir Dis 142: 13511358.

8. Moffatt MF, Kabesch M, Liang L, Dixon AL, Strachan D, et al. (2007) Genetic variants regulating ORMDL3 expression contribute to the risk of childhood asthma. Nature 448: 470-473.

9. Swarr DT, Hakonarson H (2010) Unraveling the complex genetic underpinnings of asthma and allergic disorders. Curr Opin Allergy Clin Immunol 10: $434-442$.

10. Chanock SJ, Manolio T, Boehnke M, Boerwinkle E, Hunter DJ, et al. (2007) Replicating genotype-phenotype associations. Nature 447: 655-660.

11. Daley D, Lemire M, Akhabir L, Chan-Yeung M, He JQ et al. (2009) Analyses of associations with asthma in four asthma population samples from Canada and Australia. Hum Genet 125: 445-459.

12. Hancock DB, Romieu I, Shi M, Sienra-Monge JJ, Wu H, et al. (2009) Genomewide association study implicates chromosome 9q21.31 as a susceptibility locus for asthma in mexican children. PLoS Genet 5: e1000623.

13. Himes BE, Hunninghake GM, Baurley JW, Rafaels NM, Sleiman P, et al. (2009) Genome-wide association analysis identifies PDE4D as an asthma-susceptibility gene. Am J Hum Genet 84: 581-593.

14. Mathias RA, Grant AV, Rafaels N, Hand T, Gao L, et al. (2010) A genomewide association study on African-ancestry populations for asthma. J Allergy Clin Immunol 125: 336-346.

15. Sleiman PM, Flory J, Imielinski M, Bradfield JP, Annaiah K, et al. (2010) Variants of DENND1B associated with asthma in children. N Engl J Med 362: 36-44.

16. Li X, Howard TD, Zheng SL, Haselkorn T, Peters SP, et al. (2010) Genomewide association study of asthma identifies RAD50-IL13 and HLA-DR/DQ regions. J Allergy Clin Immunol 125: 328-335.

17. Moffatt MF, Gut IG, Demenais F, Strachan DP, Bouzigon E, et al. (2010) A large-scale, consortium-based genomewide association study of asthma. N Engl J Med 363: 1211-1221.
(DOC)

Table S7 Coverage of candidate genes on commercial arrays used in asthma GWAS in samples of European ancestry.

(DOG)

Text S1 Supplementary methods.

(DOC)

\section{Acknowledgments}

The authors want to thank Tobías Felipe for programming, and María Torres (CeGen, Santiago de Compostela Node) for her exceptional technical support with iPLEX ${ }^{\circledR}$ Gold assays. In addition, we would like to express our gratitude to the Juvenile Diabetes Research Foundation/ Wellcome Trust Diabetes and Inflammation Laboratory (JDRF/WT DIL, Cambridge Institute for Medical Research) for granting the access to the $M S 4 A 2$ re-sequencing data, and acknowledge that those who carried out the original analysis and collection of the data bear no responsibility for the analysis or interpretations of this study.

\section{Author Contributions}

Conceived and designed the experiments: MPY CF. Performed the experiments: MPY AC JC PP ISM ASP JF OAF NB JCGR JV TC. Analyzed the data: MPY CF. Contributed reagents/materials/analysis tools: CF MH JV. Wrote the paper: MPY MH CF.

18. Ferreira MA, McRae AF, Medland SE, Nyholt DR, Gordon SD, et al. (2011) Association between ORMDL3, IL1RL1 and a deletion on chromosome 17q21 with asthma risk in Australia. Eur J Hum Genet 19: 458-464.

19. Torgerson DG, Ampleford EJ, Chiu GY, Gauderman WJ, Gignoux CR, et al. (2011) Meta-analysis of genome-wide association studies of asthma in ethnically diverse North American populations. Nat Genet 43: 887-892.

20. Hirota T, Takahashi A, Kubo M, Tsunoda T, Tomita K, et al. (2011) Genomewide association study identifies three new susceptibility loci for adult asthma in the Japanese population. Nat Genet 43: 893-896.

21. Ferreira MA, Matheson MC, Duffy DL, Marks GB, Hui J, et al. (2011) Identification of IL6R and chromosome 11q13.5 as risk loci for asthma. Lancet 378: 1006-1014.

22. Haldar P, Pavord ID, Shaw DE, Berry MA, Thomas M, et al. (2008) Cluster analysis and clinical asthma phenotypes. Am J Respir Crit Care Med 178: 218224 .

23. Henderson J, Granell R, Sterne J (2009) The search for new asthma phenotypes. Arch Dis Child 94: 333-336.

24. Pino-Yanes M, Sanchez-Machin I, Cumplido J, Figueroa J, Torres-Galvan MJ, et al. (2012) IL-1 receptor-associated kinase 3 gene (IRAK3) variants associate with asthma in a replication study in the Spanish population. J Allergy Clin Immunol 129: 573-575.

25. Halapi E, Gudbjartsson DF, Jonsdottir GM, Bjornsdottir US, Thorleifsson G, et al. (2010) A sequence variant on $17 q 21$ is associated with age at onset and severity of asthma. Eur J Hum Genet 18: 902-908.

26. Ahmadi KR, Weale ME, Xue ZY, Soranzo N, Yarnall DP, et al. (2005) A singlenucleotide polymorphism tagging set for human drug metabolism and transport. Nat Genet 37: 84-89.

27. Belfer I, Buzas B, Hipp H, Dean M, Evans C, et al. (2004) Haplotype structure of inflammatory cytokines genes (IL1B, IL6 and TNF/LTA) in US Caucasians and African Americans. Genes Immun 5: 505-512.

28. Pino-Yanes M, Corrales A, Basaldúa S, Hernández A, Guerra L, et al. (2011) North African influences and potential bias in case-control association studies in the Spanish population. PLoS One 6: e18389.

29. Price AL, Patterson NJ, Plenge RM, Weinblatt ME, Shadick NA, et al. (2006) Principal components analysis corrects for stratification in genome-wide association studies. Nat Genet 38: 904-909.

30. R Development Core Team (2008) R: A language and environment for statistical computing; computing RffS, editor. Viena, Austria.

31. Wigginton JE, Cutler DJ, Abecasis GR (2005) A note on exact tests of HardyWeinberg equilibrium. Am J Hum Genet 76: 887-893.

32. Sun X, Ma SF, Wade MS, Flores C, Pino-Yanes M, et al. (2010) Functional variants of sphingosine-1-phosphate receptor 1 gene associate with asthma susceptibility. J Allergy Clin Immunol 126: 241-249. 
33. Chakraborty R, Zhong Y (1994) Statistical power of an exact test of HardyWeinberg proportions of genotypic data at a multiallelic locus. Hum Hered 44: $1-9$.

34. Salanti G, Amountza G, Ntzani EE, Ioannidis JP (2005) Hardy-Weinberg equilibrium in genetic association studies: an empirical evaluation of reporting, deviations, and power. Eur J Hum Genet 13: 840-848.

35. Balding DJ (2006) A tutorial on statistical methods for population association studies. Nat Rev Genet 7: 781-791.

36. Gonzalez JR, Armengol L, Sole X, Guino E, Mercader JM, et al. (2007) SNPassoc: an $\mathrm{R}$ package to perform whole genome association studies. Bioinformatics 23: 644-645.

37. Li Y, Willer CJ, Ding J, Scheet P, Abecasis GR (2010) MaCH: using sequence and genotype data to estimate haplotypes and unobserved genotypes. Genet Epidemiol 34: 816-834.

38. The 1000 Genomes Project Consortium (2010) A map of human genome variation from population-scale sequencing. Nature 467: 1061-1073.

39. Macgregor S, Craddock N, Holmans PA (2006) Use of phenotypic covariates in association analysis by sequential addition of cases. Eur J Hum Genet 14: 529 534.

40. Pruim RJ, Welch RP, Sanna S, Teslovich TM, Chines PS, et al. (2010) LocusZoom: regional visualization of genome-wide association scan results. Bioinformatics 26: 2336-2337.

41. Storey JD, Tibshirani R (2003) Statistical significance for genomewide studies. Proc Natl Acad Sci USA 100: 9440-9445.

42. Ward LD, Kellis M (2012) HaploReg: a resource for exploring chromatin states, conservation, and regulatory motif alterations within sets of genetically linked variants. Nucleic Acids Res 40: D930-934.

43. Vercelli D (2008) Discovering susceptibility genes for asthma and allergy. Nat Rev Immunol 8: 169-182.

44. Gelfand EW (2008) Is asthma in childhood different from asthma in adults? Why do we need special approaches to asthma in children? Allergy Asthma Proc 29: 99-102.

45. Lasky-Su J, Lyon HN, Emilsson V, Heid IM, Molony C, et al. (2008) On the replication of genetic associations: timing can be everything! Am J Hum Genet 82: 849-858.

46. Bouzigon E, Corda E, Aschard H, Dizier MH, Boland A, et al. (2008) Effect of $17 q 21$ variants and smoking exposure in early-onset asthma. N Engl J Med 359: 1985-1994.

47. Castro-Giner F, de Cid R, Gonzalez JR, Jarvis D, Heinrich J, et al. (2010) Positionally cloned genes and age-specific effects in asthma and atopy: an international population-based cohort study (ECRHS). Thorax 65: 124-131.

48. Barbee RA, Kaltenborn W, Lebowitz MD, Burrows B (1987) Longitudinal changes in allergen skin test reactivity in a community population sample. J Allergy Clin Immunol 79: 16-24.
49. Boulet LP, Turcotte H, Laprise C, Lavertu C, Bedard PM, et al. (1997) Comparative degree and type of sensitization to common indoor and outdoor allergens in subjects with allergic rhinitis and/or asthma. Clin Exp Allergy 27: $52-59$.

50. Nicolae DL, Ober C (2009) (Too) great expectations: the challenges in replicating asthma disease genes. Am J Respir Crit Care Med 179: 1078-1079.

51. Pulit SL, Voight BF, de Bakker PI (2010) Multiethnic genetic association studies improve power for locus discovery. PLoS One 5: e12600.

52. Manolio TA, Collins FS, Cox NJ, Goldstein DB, Hindorff LA, et al. (2009) Finding the missing heritability of complex diseases. Nature 461: 747-753.

53. Baye TM, Butsch Kovacic M, Biagini Myers JM, Martin LJ, Lindsey M, et al. (2011) Differences in candidate gene association between European ancestry and African American asthmatic children. PLoS One 6: e16522.

54. Botigue LR, Henn BM, Gravel S, Maples BK, Gignoux CR, et al. (2013) Gene flow from North Africa contributes to differential human genetic diversity in southern Europe. Proc Natl Acad Sci USA, in press.

55. Hindorff LA, Sethupathy P, Junkins HA, Ramos EM, Mehta JP, et al. (2009) Potential etiologic and functional implications of genome-wide association loci for human diseases and traits. Proc Natl Acad Sci USA 106: 9362-9367.

56. Contopoulos-Ioannidis DG, Manoli EN, Ioannidis JP (2005) Meta-analysis of the association of beta2-adrenergic receptor polymorphisms with asthma phenotypes. J Allergy Clin Immunol 115: 963-972.

57. Finkelstein Y, Bournissen FG, Hutson JR, Shannon M (2009) Polymorphism of the ADRB2 gene and response to inhaled beta-agonists in children with asthma: a meta-analysis. J Asthma 46: 900-905.

58. Simpson A, John SL, Jury F, Niven R, Woodcock A, et al. (2006) Endotoxin exposure, CD14, and allergic disease: an interaction between genes and the environment. Am J Respir Crit Care Med 174: 386-392.

59. Michel S, Liang L, Depner M, Klopp N, Ruether A, et al. (2010) Unifying candidate gene and GWAS Approaches in Asthma. PLoS One 5: e13894.

60. Granada M, Wilk JB, Tuzova M, Strachan DP, Weidinger S, et al. (2012) A genome-wide association study of plasma total IgE concentrations in the Framingham Heart Study. J Allergy Clin Immunol 129: 840-845.

61. Galanter JM, Torgerson D, Gignoux CR, Sen S, Roth LA, et al. (2011) Cosmopolitan and ethnic-specific replication of genetic risk factors for asthma in 2 Latino populations. J Allergy Clin Immunol 128: 37-43.

62. Rogers AJ, Raby BA, Lasky-Su JA, Murphy A, Lazarus R, et al. (2009) Assessing the reproducibility of asthma candidate gene associations, using genome-wide data. Am J Respir Crit Care Med 179: 1084-1090.

63. Wu H, Romieu I, Shi M, Hancock DB, Li H, et al. (2010) Evaluation of candidate genes in a genome-wide association study of childhood asthma in Mexicans. J Allergy Clin Immunol 125: 321-327. 